\title{
Biomarkers and Dynamic Cerebral Autoregulation of Obstructive Sleep Apnea-Hypopnea Syndrome
}

\author{
Yanan Zhang' \\ Xiaodan $\mathrm{Wu}^{\prime}$ \\ Qingqing Sun' \\ Qi Tang' \\ Zhen-Ni Guo ${ }^{2}$ \\ Zan Wang' \\ Yi Yang ${ }^{2}$
}

'Department of Neurology and Neuroscience Center, the First Hospital of Jilin University, Changchun, People's Republic of China; ${ }^{2}$ Department of Neurology and Neuroscience Center, Clinical Trial and Research Center for Stroke, the First Hospital of Jilin University, Changchun, People's Republic of China
Correspondence: Zan Wang Department of Neurology and Neuroscience Center, the First Hospital of Jilin University, Changchun, People's Republic of China

Email wangzan@jlu.edu.cn;

wangzanprof@I63.com

Yi Yang

Department of Neurology and Neuroscience Center, Clinical Trial and Research Center for Stroke, the First Hospital of Jilin University, Changchun, People's Republic of China Email yang_yi@jlu.edu.cn; doctoryangyi@I63.com
Objective: Patients with obstructive sleep apnea-hypopnea syndrome (OSAHS) have impaired dynamic cerebral autoregulation (dCA). This study aimed to explore whether serum levels of apolipoprotein B-100 (ApoB-100), fibronectin (FN), and ceruloplasmin (CP) were related to impaired dCA in OSAHS.

Methods: A total of 90 patients with OSAHS from our database management system were enrolled and further divided into three subgroups according to the apnea-hypopnea index (AHI) using polysomnography results: mild $(5 \leq \mathrm{AHI} \leq 15)$, moderate $(15<\mathrm{AHI} \leq 30)$, and severe OSAHS (AHI > 30), with 30 patients in each group. Thirty sex- and age-matched healthy controls were recruited for this study. The serum levels of ApoB-100, FN, and CP were measured by enzyme-linked immunosorbent assays. dCA was assessed by analyzing the phase difference (PD) using transfer function analysis.

Results: Serum levels of ApoB-100, FN, and CP were significantly higher in the mild, moderate, and severe OSAHS groups than that in the control group $(P<0.001$, respectively). The average PD of the moderate and severe OSAHS groups was lower than that of the control group ( $P=0.001$ and $P<0.001$, respectively). Receiver operating curve analysis revealed that ApoB-100, FN, and $\mathrm{CP}$ might be able to distinguish patients with OSAHS from healthy individuals (area under the curve $=0.959$ [95\% CI 0.92-1.00], 0.987 [95\% CI 0.96-1.01], 0.982 [95\% CI 0.96-1.00]), respectively, $P<0.001$ ). The average PD was linearly correlated with the serum levels of ApoB-100, FN, and CP in patients with OSAHS. Multivariable analysis showed that $\mathrm{FN}$ and arousal index in polysomnography were associated with impaired average $\mathrm{PD}(P<0.001$ and $P=0.025$, respectively).

Conclusion: Serum levels of ApoB-100, FN, and CP increased in patients with OSAHS. dCA was compromised in patients with OSAHS and was positively correlated with ApoB$100, \mathrm{FN}$, and CP serum levels, and FN serum levels and arousal index in polysomnography were independently associated with impaired dCA.

Keywords: obstructive sleep apnea-hypopnea syndrome, apolipoprotein B-100, fibronectin, ceruloplasmin, dynamic cerebral autoregulation

\section{Introduction}

Obstructive sleep apnea-hypopnea syndrome (OSAHS) is a common disease characterized by a decrease or cessation of airflow due to repeated collapse and obstruction of the upper airway and results in recurrent oxyhemoglobin desaturation and sleep fragmentation, ${ }^{1}$ which triggers the development of systemic inflammation, ${ }^{2}$ oxidative stress, ${ }^{3}$ endothelial dysfunction, ${ }^{4}$ and metabolic syndrome. ${ }^{5}$ Studies have found that 
dynamic cerebral autoregulation (dCA) is impaired in patients with OSAHS, which may play an important role in the predisposition to stroke in these patients. ${ }^{6}$ Cerebral autoregulation (CA) is the intrinsic ability of the brain to maintain adequate cerebral perfusion in the presence of blood pressure changes. dCA can respond to real-time changes in blood pressure within seconds, allowing continuous measurement of CA and wave-by-wave analysis of hemodynamics. ${ }^{7}$ The dCA mechanism is complex and includes myogenic, metabolic, endothelial, and neurogenic mechanisms. ${ }^{8}$

The diagnosis and severity of OSAHS is usually based on polysomnography (PSG), which is expensive and cumbersome. ${ }^{9}$ Therefore, it is important to identify predictive biomarkers of OSAHS. ${ }^{10}$ Studies have found that apolipoprotein B-100 (ApoB-100), fibronectin (FN), and ceruloplasmin $(\mathrm{CP})$ are overexpressed in patients with OSAHS and correlate with the severity of disease. ${ }^{5}$ OSAHS could cause metabolic dysfunction and abnormal lipid metabolism; in such cases, the utilization rate of lipids decreases, resulting in an increase in plasma levels. ApoB-100 can induce macrophages to take in cholesterol and form foam cells, which can cause atherosclerosis. ${ }^{11}$ In addition, hypoxia could promote the synthesis and secretion of FN in myofibroblasts in patients with OSAHS, ${ }^{12}$ and acute inflammation interferes with the normal degradation process of $\mathrm{CP}$, resulting in increased serum levels of CP. ${ }^{13}$ FN may affect platelet adhesion on fibrin and promote thrombosis indirectly. ${ }^{14} \mathrm{CP}$ is an acute inflammatory response protein, which could oxidize and deform lowdensity lipoprotein (LDL) and promote vascular lesions. The increasing serum levels of CP can result in atherosclerotic plaque rupture. ${ }^{15} \mathrm{ApoB}-100, \mathrm{FN}$, and $\mathrm{CP}$ are related to several metabolic pathways, such as the lipid and vascular metabolic pathways, ${ }^{16}$ suggesting that they may facilitate the onset and progression of atherogenesis and likely affect dCA.

In this study, we assessed $\mathrm{dCA}$ and the serum levels of ApoB-100, FN, and CP from patients with OSAHS and explored the relationship among dCA, serum marker, and OSAHS, providing more evidence for the evaluation and diagnosis of OSAHS.

\section{Methods}

The study was approved by the ethics committee of the First Hospital of Jilin University in accordance with the guidelines of the Declaration of Helsinki (1964). All participants and guardians signed written informed consent forms.

\section{Participants}

Patients with OSAHS who were already scheduled for PSG were recruited from the database management system of Department of Neurology, First Hospital of Jilin University, from March 2019 to March 2020. Patients were classified according to their apnea-hypopnea index (AHI) scores as mild $(5 \leq \mathrm{AHI} \leq 15)$, moderate $(15<\mathrm{AHI}$ $\leq 30$ ), or severe (AHI $>30)$. There were 90 OSAHS patients with good bilateral temporal window penetration from the database management system enrolled (30 with mild OSAHS, 30 with moderate OSAHS, and 30 with severe OSAHS). Thirty sex- and age-matched healthy controls were recruited from the same region according to their PSG results.

The inclusion criteria for the different groups were as follows: (1) Control group: age- and sex-matched healthy volunteers without sleep disorder in PSG; (2) OSAHS groups: met the diagnostic criteria of mild, moderate, and severe OSAHS according to the International Classification of Sleep Disorders 3rd edition; (3) age between 18 and 65 years; and (4) good bilateral temporal window penetration (could detect cerebral hemispheric blood flow signal and spectrum through the temporal window for 10 minutes).

The exclusion criteria included (1) combination of other sleep disorders except for OSAHS according to PSG; (2) epilepsy, psychiatric disorders, drug abuse, suspicion of anxiety [7-item Hamilton Anxiety Rating Scale $>8$ ] or depression [7-item Hamilton Depression Rating Scale $>$ 8]; (3) arrhythmia, hyperthyroidism, and other hemodynamic factors; (4) intracranial and extracranial vascular stenosis or occlusion diagnosed by vascular ultrasound; and (5) inability to cooperate with the questionnaire survey.

\section{Collection of Clinical Data}

Clinical evaluation included sex, age, medical history, neurological examination, and magnetic resonance imaging. Questionnaires were completed, and medical histories were obtained before the subjects underwent sleep studies. Daytime sleepiness was assessed using the Epworth Sleepiness Scale (ESS).

\section{Polysomnography}

All subjects were monitored for at least $8 \mathrm{~h}$ at the sleep center of our hospital using PSG (Compumedics, Abbotsford, Australia). PSG results were analyzed by professional sleep technicians with PSG technologist 
certification by referring to the revised interpretation criteria of sleep stages and related events issued by the American Academy of Sleep Medicine version 2.3. Apnea was defined as a $\geq 90 \%$ decrease in airflow for more than $10 \mathrm{~s}$; hypopnea was defined as a $\geq 30 \%$ decrease in airflow for $10 \mathrm{~s}$ associated with a decrease in oxygen saturation for $\geq 3 \%$, or electroencephalogram arousal. AHI was defined as the number of apnea or hypopnea events per hour during sleep.

\section{ApoB-100, FN, and CP Serum Level Detection}

Blood samples and dCA from all participants were collected between 8:00-11:00 am. Serum samples were gauged using morning fasting venous blood samples and centrifuged at $3000 \mathrm{rpm}$ for $10 \mathrm{~min}$. The serum was stored at $-20^{\circ} \mathrm{C}$ before further use. Enzyme-linked immunosorbent assays (ELISA; Enzyme Immunoassay Kit, Chi Mingrui Biological Technology Co., Ltd, Shanghai, China) were used to measure the serum levels of ApoB$100, \mathrm{FN}$, and CP.

\section{Dynamic Cerebral Autoregulation Measurement and Analysis}

Participants were instructed to avoid alcohol, nicotine, and caffeine intake and exercise for at least $12 \mathrm{~h}$ before the measurement. The measurements were performed in a quiet, dedicated laboratory. First, the subjects had their baseline arterial blood pressure (Omron 711; Omron, Kyoto, Japan) and heart rate (HR) measured. ${ }^{17}$ Then, beatto-beat arterial blood pressure (Finometer Model 1; Finapres Medical Systems, Enschede, Netherlands) and continuous bilateral middle cerebral artery blood flow velocity (MultiDop X2; DWL, Sipplingen, Germany) were recorded spontaneously for $10 \mathrm{~min}$. The measurement data were then stored for further dCA examination analysis. $^{18}$ End-tidal $\mathrm{CO}_{2}$ was monitored using a capnography with a facemask attached to the nasal cannula. All measurements were performed by an experienced operator.

Recorded data were processed using MATLAB software (MathWorks, Natick, MA). dCA analysis was performed using transfer function analysis (TFA). TFA between the arterial blood pressure and cerebral blood flow velocity was calculated as the quotient of the crossspectrum of the two signals and the auto-spectrum of arterial blood pressure in the low-frequency domain
(0.06-0.12 Hz) to obtain the frequency-dependent estimates of phase difference (PD), where the derived parameters are considered to be the most relevant to autoregulation hemodynamics. ${ }^{19} \mathrm{~A}$ decreased PD represents impaired dCA. Coherence was calculated to estimate the reliability of the relationship between the two signals in the frequency domain, and statistical analysis was later performed only if the coherence of the parameters was $>$ $0.5 .^{20}$

\section{Statistical Analysis}

SPSS software (version 23.0; IBM, Armonk, NY) was used for statistical analysis. The Shapiro-Wilk test was used to assess the normal distribution of continuous variables. Data with a normal distribution (including age, body mass index [BMI], mean arterial blood pressure [MAP], $\mathrm{HR}$, ESS, ApoB-100, FN, CP, PD, end-tidal $\mathrm{CO}_{2}$ $\left[\mathrm{ETPCO}_{2}\right]$, total sleep time [TST], sleep onset latency [SL], sleep efficiency [SE], rapid eye movement [REM], stage 1 non-REM [stage N1], stage 2 NREM [stage N2], stage 3 NREM [stage N3], arousal index, AHI, mean $\mathrm{SaO}_{2}$, minimum $\mathrm{SaO}_{2}$ ) were expressed as mean and standard deviation, whereas data with skewed distribution were expressed as medians with interquartile range. Categorical data (sex, hypertension, and hyperlipidemia) were expressed as absolute values and percentages. Oneway analysis of variance or the Kruskal-Wallis $H$-test was used to compare differences between multiple groups of independent samples (age, BMI, MAP, HR, ESS, ApoB100, FN, CP, PD, ETPCO 2 , TST, SL, SE, REM, stage N1, stage $\mathrm{N} 2$, stage $\mathrm{N} 3$, arousal index, AHI, mean $\mathrm{SaO}_{2}$, minimum $\mathrm{SaO}_{2}$ of mild, moderate, and severe OSAHS and control groups) based on the data distribution. Receiver operating characteristic (ROC) curves were used to determine the sensitivity, specificity, and cutoff point of ApoB-100, FN, and CP serum levels to predict OSAHS based on the Youden index. The correlation between PD and ApoB-100, FN, and CP serum levels was examined using the non-parametric Spearman correlation test. The correlation between ESS, sleep latency in PSG, and apoB-100, FN, and CP serum levels were examined using the non-parametric Spearman correlation test. Univariate and multivariate linear regression were used to assess the association between PD and clinical parameters including sex, age, BMI, MAP, HR, hypertension, hyperlipidemia, ETPCO 2 , ApoB-100, FN, CP, TST, SL, SE, arousal index, stage N1, stage N2, stage N3, REM, AHI, mean $\mathrm{SaO}_{2}$, and minimum $\mathrm{SaO}_{2}$. In the post-hoc analysis, 
the Bonferroni method was used to calculate the adjusted $P$-value. Statistical significance was set at $P<0.05$.

\section{Results}

\section{Baseline Characteristics}

A total of 120 participants were enrolled in this study, including 30 mild OSAHS patients, 30 moderate OSAHS patients, 30 severe OSAHS patients, and 30 controls. There were no significant differences in sex, age, MAP, $\mathrm{HR}$, hypertension, hyperlipidemia, or $\mathrm{ETPCO}_{2}$ among the study groups. BMI was significantly higher in the severe OSAHS group compared to the control and mild OSAHS groups $(P<0.05)$. ESS scores in patients with mild, moderate, and severe OSAHS were significantly higher than those in the control group $(P<0.001)$ (Table 1$)$. The PSG parameters of all groups are presented in Table 2.

\section{Comparison of ApoB-100, FN and CP Serum Levels in All Groups}

ApoB-100, FN, and CP serum levels in patients with mild, moderate, and severe OSAHS were significantly higher than those in the control group $(P<0.001)$, and ApoB$100, \mathrm{FN}$, and $\mathrm{CP}$ serum levels declined gradually with the severity of OSAHS (Table 1 and Figure 1).

\section{Comparison of dCA Parameters in All Groups}

There were no significant differences between the right and left dCA parameters (PD) between groups. Therefore, average values were used in the subsequent analysis. The average PD of patients in the moderate OSAHS group $\left(39.05 \pm 10.97^{\circ}\right)$ and severe OSAHS group (31.14 \pm $17.45^{\circ}$ ) were lower than that of the control group (52.37 $\left.\pm 9.39^{\circ}, P=0.001, P<0.001\right)$ or mild OSAHS group $\left(48.22 \pm 12.27^{\circ}, P=0.041, P<0.001\right)$ (Table 1 and Figure 2).

\section{Discriminative Power of ApoB-I00, FN, and CP Serum Values to Distinguish}

\section{Between OSAHS and Non-OSAHS}

In the ROC analysis, the areas under the curve (AUCs) of ApoB-100, FN, and CP were 0.959 (95\% confidence interval [CI]: 0.92 to $1.00, P<0.001), 0.987$ (95\% CI: 0.96 to 1.01, $P<0.001$ ), and 0.982 (95\% CI: 0.96 to $1.00, P<$ 0.001 ), respectively. The optimal cutoff value for ApoB100 was $0.594 \mathrm{~g} / \mathrm{L}$ (sensitivity, 95.6\%; specificity, 96.7\%),
$0.735 \mathrm{~g} / \mathrm{L}$ for FN (sensitivity, 96.7\%; specificity, 100.0\%), and $0.731 \mathrm{~g} / \mathrm{L}$ for $\mathrm{CP}$ (sensitivity, $94.4 \%$; specificity, 96.7\%) (Figure 3).

\section{Correlation Analysis for ESS, Sleep Latency, and ApoB-100, FN, and CP}

\section{Serum Levels in OSAHS}

In the Spearman correlation analysis, the ESS in patients with OSAHS was weakly correlated with ApoB-100 ( $\mathrm{r}=$ $0.231, P=0.028), \mathrm{FN}(\mathrm{r}=0.310, P=0.003)$, and $\mathrm{CP}(\mathrm{r}=$ $0.263, P=0.012)$. Sleep latency in patients with OSAHS was not correlated with ApoB-100 $(\mathrm{r}=0.026, P=0.808)$, $\mathrm{FN}(\mathrm{r}=0.151, P=0.155)$, or $\mathrm{CP}(\mathrm{r}=0.037, P=0.727)$.

\section{Correlation Analysis for Average PD and ApoB-100, FN, and CP Serum Levels in OSAHS}

In the Spearman correlation analysis, the average PD values in patients with OSAHS were positively correlated with ApoB-100 ( $\mathrm{r}=0.695, P<0.001)$, FN ( $\mathrm{r}=0.850, P<$ $0.001)$, and $\mathrm{CP}(\mathrm{r}=0.792, P<0.001)$ (Figure 4$)$.

\section{Univariable and Multivariable Analysis for Factors Affecting dCA}

The clinical parameters used in the univariate and multivariate analyses are presented in Table 3. In the univariable model, average PD was correlated with ApoB-100 $(P<0.001)$, FN $(P<0.001), \mathrm{CP}(P<0.001)$, SL $(P=0.032)$, stage N1 $(P=$ $0.022)$, arousal index $(P<0.001)$, AHI $(P<0.001)$, mean $\mathrm{SaO}_{2}(P<0.001)$, and minimum $\mathrm{SaO}_{2}(P<0.001)$.

The clinical parameters with $P \leq 0.1$ in the univariable analysis (ApoB-100, FN, CP, SL, stage N1, arousal index, AHI, mean $\mathrm{SaO}_{2}$, and minimum $\mathrm{SaO}_{2}$ ) were included in the multivariable model. FN $(P<0.001)$ and arousal index $(P=$ $0.025)$ were independently associated with impaired average PD.

\section{Discussion}

This study found that serum levels of ApoB-100, FN, and $\mathrm{CP}$ increased in patients with OSAHS. dCA was compromised in patients with OSAHS and was positively correlated with ApoB-100, FN, and CP serum levels, and FN serum levels and arousal index in polysomnography were independently associated with impaired dCA.

Obstructive sleep apnea (OSA) is a highly prevalent medical condition that remains underdiagnosed. The 
Table I Clinical Characteristics, ApoB-100, FN, CP Serum Levels and Phase Difference in the Patients with Mild, Moderate, Severe OSAHS and Controls

\begin{tabular}{|c|c|c|c|c|c|}
\hline & Controls $(n=30)$ & Mild OSAHS $(n=30)$ & Moderate OSAHS $(n=30)$ & Severe OSAHS $(n=30)$ & $P$ \\
\hline Male, n (\%) & $21(70.0)$ & $18(60.0)$ & $22(73.3)$ & $24(80.0)$ & 0.388 \\
\hline Age (years) & $48.1 \pm 10.4$ & $48.3 \pm 10.3$ & $49.9 \pm 11.7$ & $49.3 \pm 9.4$ & 0.896 \\
\hline BMI $\left(\mathrm{kg} / \mathrm{m}^{2}\right)$ & $24.00 \pm 3.43$ & $24.47 \pm 3.41$ & $25.48 \pm 2.45$ & $26.54 \pm 2.51$ & $0.007^{\mathrm{a}}$ \\
\hline $\mathrm{MAP}(\mathrm{mmHg})$ & $85.03 \pm 14.90$ & $84.23 \pm \mid 2.21$ & $85.93 \pm 11.30$ & $86.77 \pm 8.66$ & 0.860 \\
\hline Heart Rate (beats/min) & $65.1 \pm 7.7$ & $68.7 \pm 8.7$ & $66.6 \pm 7.3$ & $68.7 \pm 8.6$ & 0.238 \\
\hline Hypertension, n (\%) & $4(13.3)$ & $6(20.0)$ & $8(26.7)$ & $8(26.7)$ & 0.540 \\
\hline Hyperlipidemia, n (\%) & $5(16.7)$ & $6(20.0)$ & $9(30.0)$ & $7(23.3)$ & 0.643 \\
\hline Epworth sleepiness scale & $2.4 \pm 1.0$ & $6.7 \pm 3.8$ & $11.9 \pm 5.1$ & $11.1 \pm 5.0$ & $<0.001^{a}$ \\
\hline ApoB-100 (g/L) & $0.470 \pm 0.093$ & $1.515 \pm 0.392$ & $1.143 \pm 0.204$ & $0.947 \pm 0.350$ & $<0.001^{a}$ \\
\hline $\mathrm{FN}(g / \mathrm{L})$ & $0.483 \pm 0.109$ & $1.629 \pm 0.313$ & $1.260 \pm 0.240$ & $1.098 \pm 0.302$ & $<0.001^{\mathrm{a}}$ \\
\hline $\mathrm{CP}(\mathrm{g} / \mathrm{L})$ & $0.496 \pm 0.127$ & $1.665 \pm 0.351$ & $1.178 \pm 0.276$ & $1.032 \pm 0.257$ & $<0.001^{a}$ \\
\hline Phase difference (degree) & $52.37 \pm 9.39$ & $48.22 \pm 12.27$ & $39.05 \pm 10.97$ & $31.14 \pm 17.45$ & $<0.001^{a}$ \\
\hline End-tidal $\mathrm{CO}_{2}(\mathrm{mmHg})$ & $35.52 \pm 2.52$ & $35.41 \pm 2.20$ & $35.38 \pm 3.14$ & $35.72 \pm 2.94$ & 0.963 \\
\hline
\end{tabular}

Note: ${ }^{a} P$ value $<0.05$ (statistically different).

Abbreviations: BMI, body mass index; MAP, mean arterial pressure; ApoB-100, apolipoprotein B-100; FN, fibronectin; CP, ceruloplasmin.

American Academy of Sleep Medicine reports that blood biomarkers may be useful in identifying individuals at risk for OSA. ${ }^{21}$ Fleming et al found that concurrent elevations of glycated hemoglobin (HbAlc), C-reactive protein (CRP), and erythropoietin (EPO) levels may be useful as an OSA screening tool and correlate with OSA severity. ${ }^{22}$ Li et al suggested that patients with OSA, particularly moderate-severe OSA and those with $\mathrm{BMI} \geq 30$, are significantly more likely to have elevated levels of CRP/highsensitivity CRP. ${ }^{23}$ Gabryelska et al described that serum hypoxia-inducible factor 1 (HIF-1 $\alpha$ ) was increased in OSA patients, ${ }^{24}$ which was caused by intermittent nocturnal hypoxia. ${ }^{25}$ Furthermore, single-night continuous positive airway pressure (CPAP) therapy seems to be insufficient to affect the increased level of the protein. ${ }^{26} \mathrm{Lu}$ et al observed decreased levels of HIF- $1 \alpha$ following 2 months of CPAP treatment compared to baseline results. ${ }^{27}$ Many previous studies have shown that OSAHS is associated with dyslipidemia. ${ }^{28-30}$ The abnormal metabolism of ApoB-100 is an important component of dyslipidemia and is dynamically related to overproduction of very lowdensity lipoprotein (VLDL) and ApoB-100, decreased catabolism of apolipoprotein B particles, and accelerated catabolism of high-density lipoprotein (HDL) particles. ${ }^{31}$ The utilization of lipids could affect the metabolic pool of intercellular ApoB-100. Chronic intermittent hypoxia can cause metabolic dysfunction and abnormal lipid metabolism, leading to decreased utilization of lipids and an increase in plasma levels. $\mathrm{FN}$ is a protein marker for the activation of endothelial cells. ${ }^{32}$ The mechanism of increased FN serum levels in patients with OSAHS is that hypoxia increases FN synthesis at the transcriptional level and promotes the secretion of FN into the extracellular fluid. ${ }^{12}$ A study has found that the main degradation enzymes of FN, including matrix metallopeptidase- 2 and matrix metallopeptidase-9, are increased in patients with OSAHS ${ }^{33}$ which indicates that the increase in extracellular FN is not caused by a decrease in degradation and suggests that hypoxia could promote the synthesis and secretion of $\mathrm{FN}$ in myofibroblasts. In patients with OSAHS, glycolipid shedding on the cell surface increases because of acute inflammation, which leads to an abnormal increase of sialic acid in blood. Activated sialyltransferase degrades and desialylates $\mathrm{CP}$, thus affecting its normal degradation process and resulting in an increase in the serum level of CP. ${ }^{13}$ We are studying some inflammatory factors, including immunoglobulin $\lambda$ light chain $(\lambda$ IgLC), filaggrin (FLG), transthyretin (TTR) and will further add them in the follow-up study.

Some studies have shown that changes in these serum markers show an upward trend with the gradual aggravation of the severity of OSAHS, ${ }^{16}$ but not all of them. Gamez et al found that antithrombin and thrombin sensitizing protein-1 serum levels decreased in the moderate group and thrombin sensitizing protein-1 decreased in the severe group with low protein expression. CP serum levels increased in the moderate group and decreased significantly in the severe group. ${ }^{34}$ There was also incomplete aggravation in our research 
Table 2 Polysomnography Parameters in the Patients with Mild, Moderate, Severe OSAHS and Controls

\begin{tabular}{|c|c|c|c|c|c|}
\hline & Controls $(n=30)$ & Mild OSAHS $(n=30)$ & Moderate OSAHS $(n=30)$ & Severe OSAHS $(n=30)$ & $P$ \\
\hline Total Sleep Time (min) & $390.40 \pm 33.23$ & $389.86 \pm 45.97$ & $386.14 \pm 62.17$ & $379.15 \pm 59.89$ & 0.824 \\
\hline Sleep onset latency ( $\mathrm{min}$ ) & $15.53 \pm 3.03$ & $|5.09 \pm 2.5|$ & $14.89 \pm 3.24$ & $13.75 \pm 4.02$ & 0.187 \\
\hline Sleep Efficiency (\%) & $76.43 \pm 7.92$ & $71.13 \pm 13.96$ & $62.55 \pm 18.09$ & $64.42 \pm 18.50$ & $0.002^{b}$ \\
\hline Stage NI (\%) & $4.42 \pm 1.24$ & $26.22 \pm 6.23$ & $27.32 \pm 5.83$ & $32.67 \pm 8.00$ & $<0.001^{\mathrm{b}}$ \\
\hline Stage N2 (\%) & $50.47 \pm 6.97$ & $46.65 \pm 9.73$ & $54.12 \pm 5.32$ & $45.76 \pm 5.58$ & $<0.001^{b}$ \\
\hline Stage N3 (\%) & $21.20 \pm 5.02$ & $11.60 \pm 3.13$ & $10.81 \pm 3.06$ & $10.05 \pm 2.62$ & $<0.001^{b}$ \\
\hline REM Sleep (\%) & $23.90 \pm 8.59$ & $15.54 \pm 10.40$ & $7.75 \pm 6.74$ & $11.53 \pm 9.46$ & $<0.001^{b}$ \\
\hline Arousal Index & $18.23 \pm 7.59$ & $21.47 \pm 11.06$ & $25.97 \pm 11.27$ & $44.17 \pm 21.59$ & $<0.001^{b}$ \\
\hline AHI (events/h) & $1.62 \pm 1.29$ & $9.17 \pm 2.83$ & $21.28 \pm 4.68$ & $53.89 \pm 13.86$ & $<0.001^{b}$ \\
\hline Mean $\mathrm{SaO}_{2}(\%)$ & $95.83 \pm 1.76$ & $95.03 \pm 1.50$ & $93.83 \pm 1.86$ & $90.90 \pm 4.67$ & $<0.001^{b}$ \\
\hline Minimum $\mathrm{SaO}_{2}(\%)$ & $87.90 \pm 5.36$ & $86.30 \pm 6.09$ & $80.40 \pm 9.84$ & $73.13 \pm 13.09$ & $<0.001^{b}$ \\
\hline
\end{tabular}

Note: ${ }^{b} P$ value $<0.05$ (statistically different).

Abbreviations: REM, rapid eye movement; Stage NI, stage I non-REM; Stage N2, stage 2 non-REM; Stage N3, stage 3 non-REM; AHI, Apnea-hypopnea index; SaO 2 , arterial oxygen saturation.

results; ApoB-100, FN, and CP serum levels declined gradually with the severity of OSAHS. This may reflect abnormal protein synthesis, transportation, and ubiquitination in severe or chronic OSAHS, resulting in the decline of protein function and serum levels. It may also be that when the damage from hypoxia reaches a certain degree, it does not continue, and the body gradually employs a compensatory protective mechanism to eliminate the damage so that the inflammatory-related proteins gradually decrease in serum.

The mechanism of impaired dCA in patients with OSAHS involves endothelial, metabolic, myogenic, and neurogenic theories. Carlson et al reported for the first time that the ability of acetylcholine-induced vasodilation (endothelium-dependent response) in patients with OSAHS decreased compared with age- and BMImatched subjects. ${ }^{35}$ Endothelial dysfunction is of great significance to vasomotor function. ${ }^{36}$ ApoB-100 is the main carrier of LDL, which plays an important role in the transport of endogenous cholesterol and triglycerides as well as LDL metabolism. ${ }^{35}$ It has a strong stimulating effect on the esterification of cholesterol in macrophages, which can promote the formation of foam cells, affect endothelial function, and lead to arteriosclerosis ${ }^{11,37}$ and affect dCA. FN is a medium connection between cells and stroma, participating in the promotion of cell adhesion and activation of macrophages, and is closely related to the process of angiogenesis, inflammation, tissue fibrosis, and autoregulation. ${ }^{14,38} \mathrm{CP}$ is an acute inflammatory response protein, which could cause $\mathrm{Cu}^{2+}$ to abscise and lead to oxidative modification of LDL in certain circumstances; it can also cause endothelial injury and activate monocyte- releasing factor, leading to the formation and migration of foam cells, the proliferation of vascular smooth muscle cells, and the atherosclerosis. ${ }^{16}$ Our study showed that impaired dCA was positively correlated with ApoB-100, $\mathrm{FN}$, and $\mathrm{CP}$ serum levels in patients with moderate and severe OSAHS.

Repeated hypoventilation, apnea, and frequent awakening may affect the autonomic nervous system. Long-term hypoxemia in patients with OSAHS can activate chemoreceptors in the carotid body and increase sympathetic vasoconstriction, leading to decreased cerebral blood flow. Hypercapnia stimulates central chemoreceptors in the brainstem and increases sympathetic nerve tension through a similar process. At the same time, the negative pressure in the thoracic cavity increases, which inhibits the afferent activity of the autonomic nervous system in the pulmonary system, stimulates baroreceptors, causes vasoconstriction, ${ }^{39}$ and affects $\mathrm{dCA}$.

This study has several limitations. First, we did not examine the dCA and serum levels of ApoB-100, FN, and $\mathrm{CP}$ after standard treatment. Second, the sample size of this study was small, which may have limited the analysis. Further work is required to explore other serological markers of OSAHS and to study their relationship with $\mathrm{dCA}$, providing a basis for clinical diagnosis and treatment.

\section{Conclusions}

Serum levels of ApoB-100, FN, and CP increased in patients with OSAHS. dCA was compromised in patients with OSAHS and was positively correlated with ApoB-100, FN, 


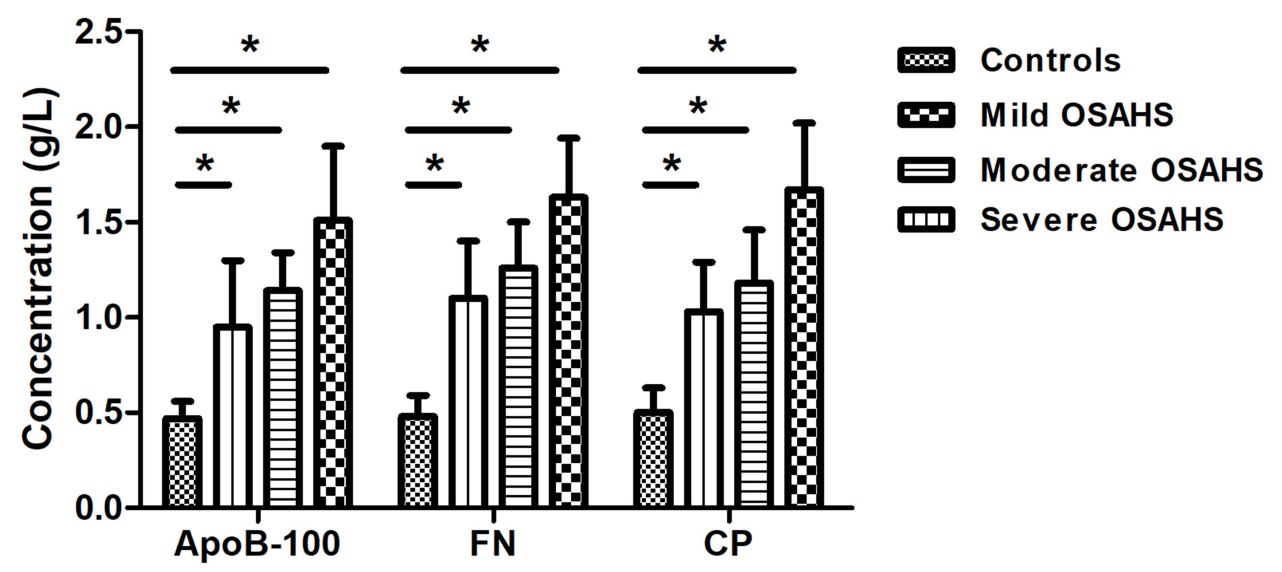

Figure I Comparison of ApoB-100, FN and CP serum levels in mild, moderate, severe OSAHS and controls. *Indicate statistically different $(P<0.05)$.

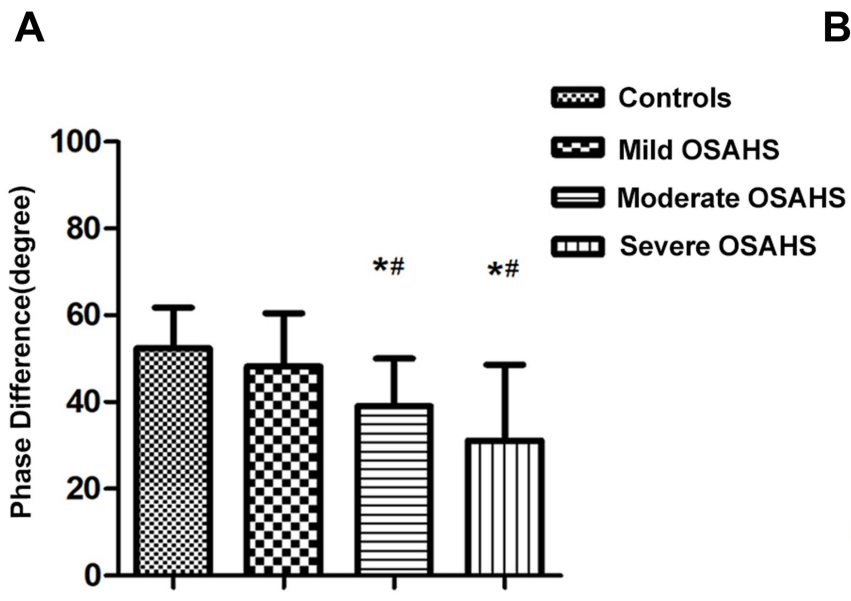

B

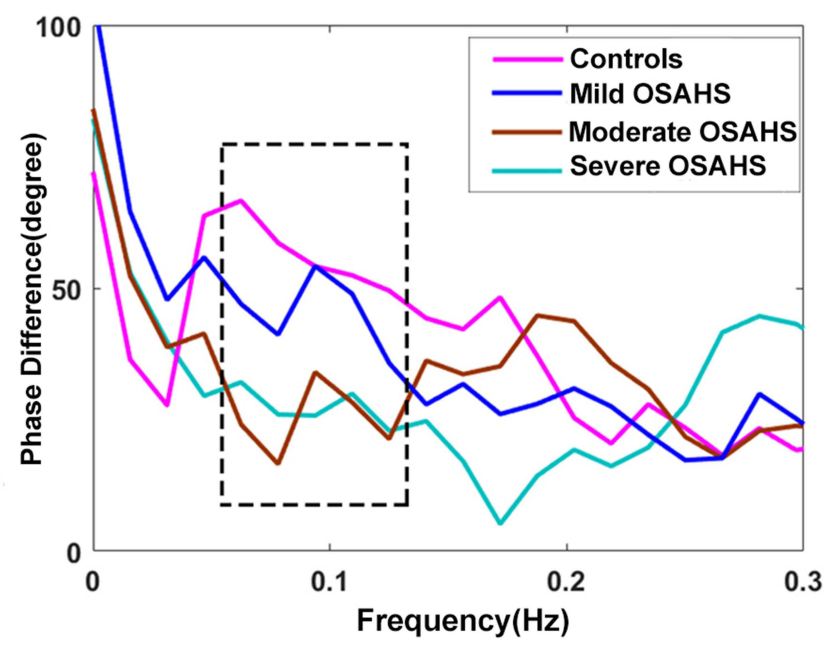

Figure 2 The autoregulatory parameter and statistical distributions in each group. Statistical distributions of average phase difference (A) and its transfer function (B) in each group. *Difference of average phase difference in moderate, severe OSAHS group and control group $(P<0.05)$. ${ }^{\#}$ Difference of average phase difference in moderate, severe OSAHS group and mild OSAHS group $(P<0.05)$. Dashed frame represent specific frequency domain $(0.06-0.21 \mathrm{~Hz})$.

A

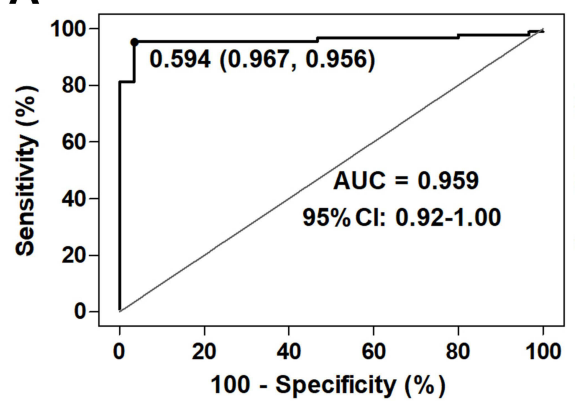

B

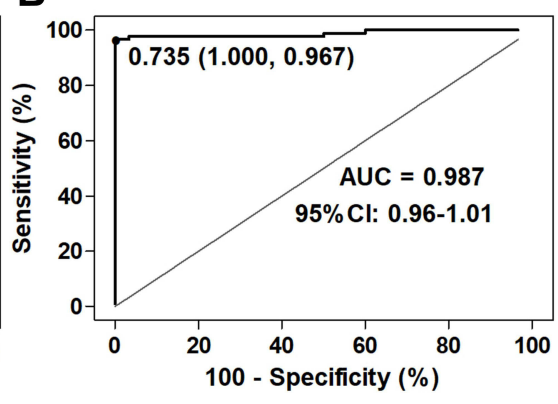

C

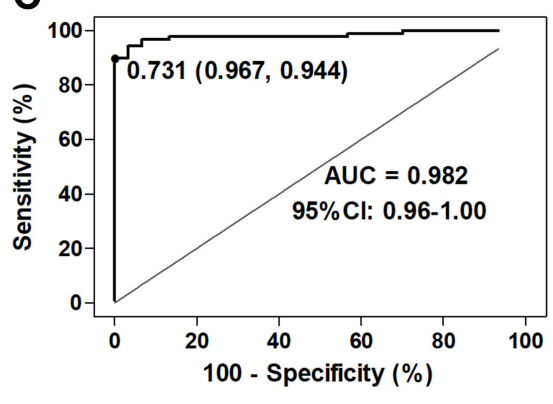

Figure 3 Receiver operating characteristic (ROC) curve for apoB-100 (A), FN (B), and CP (C) of OSAHS. Each panel shows the area under the curve (AUC), cutoff value, sensitivity, and specificity values. 
A

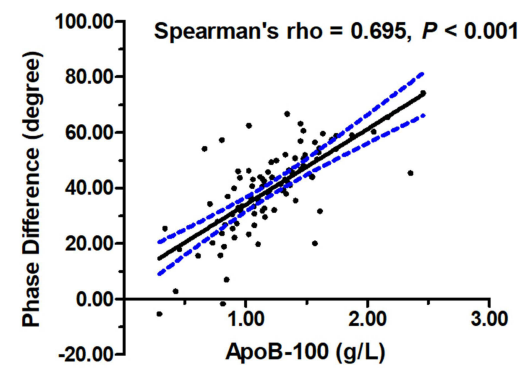

B

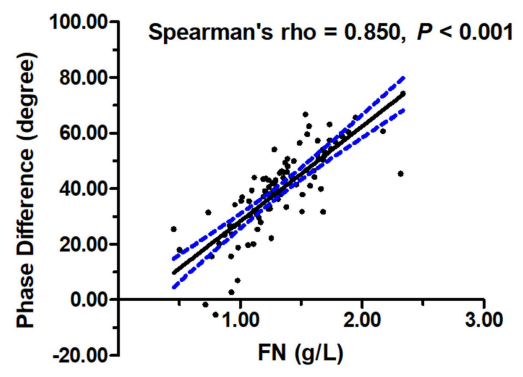

C

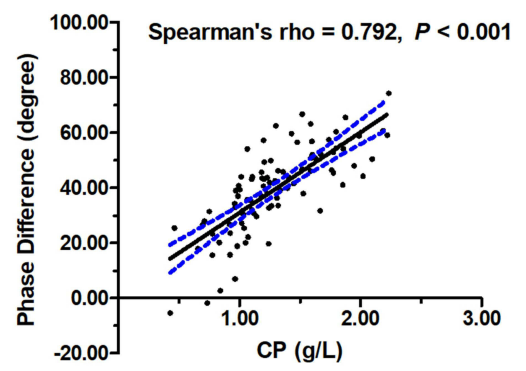

Figure 4 Relations between average phase difference and the serum levels of apoB-100 (A), FN (B), and CP (C).

Table 3 Univariable and Multivariable Analysis for the Average Phase Difference

\begin{tabular}{|c|c|c|c|c|c|c|}
\hline \multirow[t]{3}{*}{ Factors } & \multicolumn{6}{|c|}{ Average Phase Difference, Degree } \\
\hline & \multicolumn{3}{|c|}{ Univariable Analysis } & \multicolumn{3}{|c|}{ Multivariable Analysis } \\
\hline & $\beta$ & $\mathbf{P}$ & $\mathbf{R}^{2}$ & $\boldsymbol{\beta}$ & $\mathbf{P}$ & $\mathbf{R}^{2}$ \\
\hline Gender & -0.159 & 0.135 & 0.025 & & & \\
\hline Age & -0.112 & 0.293 & 0.013 & & & \\
\hline BMI & -0.145 & 0.174 & 0.021 & & & \\
\hline MAP & -0.026 & 0.807 & 0.001 & & & \\
\hline Heart Rate & -0.156 & 0.141 & 0.024 & & & \\
\hline Hypertension & -0.133 & 0.211 & 0.018 & & & \\
\hline Hyperlipidemia & -0.146 & 0.169 & 0.021 & & & \\
\hline End-tidal CO2 & -0.026 & 0.804 & 0.001 & & & \\
\hline ApoB- 100 & 0.707 & $<0.001^{a b}$ & 0.500 & 0.099 & 0.382 & \\
\hline FN & 0.800 & $<0.001^{a b}$ & 0.640 & 0.732 & $<0.001^{\mathrm{c}}$ & 0.660 \\
\hline $\mathrm{CP}$ & 0.758 & $<0.001^{a b}$ & 0.575 & 0.175 & 0.207 & \\
\hline Total Sleep Time (min) & 0.022 & 0.838 & 0.000 & & & \\
\hline Sleep onset latency $(\mathrm{min})$ & 0.152 & 0.152 & 0.023 & & & \\
\hline Sleep Efficiency (\%) & 0.226 & $0.032^{a b}$ & 0.051 & 0.021 & 0.747 & \\
\hline Stage NI (\%) & -0.242 & $0.022^{a b}$ & 0.058 & -0.010 & 0.876 & \\
\hline Stage N2 (\%) & 0.079 & 0.461 & 0.006 & & & \\
\hline Stage N3 (\%) & 0.072 & 0.498 & 0.005 & & & \\
\hline REM Sleep (\%) & 0.096 & 0.370 & 0.009 & & & \\
\hline Arousal Index & -0.471 & $<0.001^{a b}$ & 0.222 & -0.158 & $0.025^{c}$ & 0.660 \\
\hline AHI (events/h) & $-0.54 I$ & $<0.001^{a b}$ & 0.292 & 0.028 & 0.799 & \\
\hline Mean $\mathrm{SaO}_{2}(\%)$ & 0.438 & $<0.00 \mathrm{I}^{\mathrm{ab}}$ & 0.192 & 0.108 & 0.141 & \\
\hline Minimum $\mathrm{SaO}_{2}(\%)$ & 0.474 & $<0.001^{a b}$ & 0.225 & 0.084 & 0.254 & \\
\hline
\end{tabular}

Notes: ${ }^{a}$ Nominally significant values $(P<0.1)$ included in the multivariable model; ${ }^{b} P$ value $<0.05$ (statistically different); ${ }^{c}$ Associated with average phase difference.

Abbreviations: BMI, body mass index; MAP, mean arterial pressure; ApoB-100, apolipoprotein B-100; FN, fibronectin; CP, ceruloplasmin; REM, rapid eye movement; Stage NI, stage I non-REM; Stage N2, stage 2 non-REM; Stage N3, stage 3 non-REM; AHI, apnea-hypopnea index.

and CP serum levels, and FN serum levels and arousal index in polysomnography were independently associated with impaired dCA.

\section{Data Sharing Statement}

The data that support the findings of this study will be made available on request. 


\section{Acknowledgments}

The authors would like to acknowledge the following individual at Jilin University: Chunhui Wei for their technical support and Qiong Yu for the statistical analysis. Yanan Zhang and Xiaodan $\mathrm{Wu}$ are the co-first author.

\section{Funding}

The article was supported by the National Natural Science Foundation of China (Grant Number 82071489), the Foundation of the Department of Science and Technology of Jilin Province (Grant Number 20190201038JC), and the Foundation of Chinese Sleep Research Society Hansoh Project (Grant Number 2019HSB05) to ZW.

\section{Disclosure}

The authors declare no conflicts of interest for this work.

\section{References}

1. Yaggi HK, Concato J, Kernan WN, Lichtman JH, Brass LM, Mohsenin V. Obstructive sleep apnea as a risk factor for stroke and death. $N$ Engl J Med. 2005;353(19):2034-2041. doi:10.1056/ NEJMoa043104

2. Ryan S, Taylor CT, McNicholas WT. Selective activation of inflammatory pathways by intermittent hypoxia in obstructive sleep apnea syndrome. Circulation. 2005;112(17):2660. doi:10.1161/ CIRCULATIONAHA.105.556746

3. Lavie L, Lavie P. Molecular mechanisms of cardiovascular disease in OSAHS: the oxidative stress link. Eur Respir J. 2009;33 (6):1467-1484. doi:10.1183/09031936.00086608

4. Jelic S, Padeletti M, Kawut SM, et al. Inflammation, oxidative stress, and repair capacity of the vascular endothelium in obstructive sleep apnea. Circulation. 2008;117(17):2270-2278. doi:10.1161/ CIRCULATIONAHA.107.741512

5. Tanno S, Tanigawa T, Saito I, et al. Sleep-related intermittent hypoxemia and glucose intolerance: a community-based study. Sleep Med. 2014;15(10):1212-1218. doi:10.1016/j.sleep.2014.05.027

6. Nasr N, Traon AP, Czosnyka M, Tiberge M, Schmidt E, Larrue V. Cerebral autoregulation in patients with obstructive sleep apnea syndrome during wakefulness. Eur J Neurol. 2009;16(3):386-391. doi:10.1111/j.1468-1331.2008.02505.x

7. Claassen JA, Meel-van den Abeelen AS, Simpson DM, Panerai RB; International Cerebral Autoregulation Research Network (CARNet). Transfer function analysis of dynamic cerebral autoregulation: a white paper from the International Cerebral Autoregulation Research Network. J Cereb Blood Flow Metab. 2016;36 (4):665-680. doi:10.1177/0271678X15626425

8. Hamel E. Perivascular nerves and the regulation of cerebrovascular tone. J Appl Physiol. 2006;100(3):1059-1064. doi:10.1152/ japplphysiol.00954.2005

9. Madani M, Madani F. Epidemiology, pathophysiology, and clinical features of obstructive sleep apnea. Oral Maxillofac Surg Clin North Am. 2009;21(4):369-735. doi:10.1016/j.coms.2009.09.003

10. Gabryelska A, Szmyd B, Panek M, Szemraj J, Kuna P, Białasiewicz P. Serum hypoxia-inducible factor-1 $\alpha$ protein level as a diagnostic marker of obstructive sleep apnea. Pol Arch Intern Med. 2020;130(2):158-160. doi:10.20452/pamw.15104
11. Hennig B, Chow CK. Lipid peroxidation and endothelial cell injury: implications in atherosclerosis. Free Radic Biol Med. 1988;4 (2):99-106. doi:10.1016/0891-5849(88)90070-6

12. Zhang H, Yang F, Guo Y, et al. The contribution of chronic intermittent hypoxia to OSAHS: from the perspective of serum extracellular microvesicle proteins. Metabolism. 2018;85:97-108. doi:10.1016/j.metabol.2018.02.012

13. Cox C, Teknos TN, Barrios M, Brewer GJ, Dick RD, Merajver SD. The role of copper suppression as an antiangiogenic strategy in head and neck squamous cell carcinoma. Laryngoscope. 2001;111(4 Pt 1):696-701. doi:10.1097/00005537-200104000-00024

14. Wijelath ES, Rahman S, Murray J, Patel Y, Savidge G, Sobel M. Fibronectin promotes VEGF-induced CD34 cell differentiation into endothelial cells. J Vasc Surg. 2004;39(3):655-660. doi:10.1016/j. jvs.2003.10.042

15. Engström G, Lind P, Hedblad B, Stavenow L, Janzon L, Lindgärde F. Effects of cholesterol and inflammation-sensitive plasma proteins on incidence of myocardial infarction and stroke in men. Circulation. 2002;105(22):2632-2637. doi:10.1161/01. cir.0000017327.69909.ff

16. Feliciano A, Torres VM, Vaz F, et al. Overview of proteomics studies in obstructive sleep apnea. Sleep Med. 2015;16(4):437-445. doi:10.1016/j.sleep.2014.11.014

17. Lv S, Guo ZN, Jin H, et al. Compromised dynamic cerebral autoregulation in patients with epilepsy. Biomed Res Int. 2018;2018:6958476. doi:10.1155/2018/6958476.

18. Luo MY, Guo ZN, Qu Y, et al. Compromised dynamic cerebral autoregulation in patients with depression. Front Psychiatry. 2019;10:373. doi:10.3389/fpsyt.2019.00373

19. van Beek AH, Claassen JA, Rikkert MG, Jansen RW. Cerebral autoregulation: an overview of current concepts and methodology with special focus on the elderly. J Cereb Blood Flow Metab. 2008;28 (6):1071-1085. doi:10.1038/jcbfm.2008.13

20. Kuo TB, Chern CM, Sheng WY, Wong WJ, Hu HH. Frequency domain analysis of cerebral blood flow velocity and its correlation with arterial blood pressure. J Cereb Blood Flow Metab. 1998;18 (3):311-318. doi:10.1097/00004647-199803000-00010

21. Kapur VK, Auckley DH, Chowdhuri S, et al. Clinical practice guideline for diagnostic testing for adult obstructive sleep apnea: an American Academy of Sleep Medicine clinical practice guideline. J Clin Sleep Med. 2017;13(3):479-504. doi:10.5664/jcsm.6506

22. Fleming WE, Holty JC, Bogan RK, et al. Use of blood biomarkers to screen for obstructive sleep apnea. Nat Sci Sleep. 2018;10:159-167. doi:10.2147/NSS.S164488

23. Li K, Wei P, Qin Y, Wei Y. Is C-reactive protein a marker of obstructive sleep apnea?: a meta-analysis. Medicine. 2017;96(19): e6850. doi:10.1097/MD.0000000000006850

24. Gabryelska A, Karuga FF, Szmyd B, Białasiewicz P. HIF-1 $\alpha$ as a mediator of insulin resistance, T2DM, and its complications: potential links with obstructive sleep apnea. Front Physiol. 2020;11:1035. doi:10.3389/fphys.2020.01035

25. Gabryelska A, Szmyd B, Szemraj J, Stawski R, Sochal M, Białasiewicz P. Patients with obstructive sleep apnea present with chronic upregulation of serum HIF-1 $\alpha$ protein. J Clin Sleep Med. 2020;16(10):1761-1768. doi:10.5664/jcsm.8682

26. Gabryelska A, Stawski R, Sochal M, Szmyd B, Białasiewicz P. Influence of one-night CPAP therapy on the changes of HIF-1 $\alpha$ protein in OSA patients: a pilot study. J Sleep Res. 2020;29(4): e12995. doi:10.1111/jsr.12995

27. Lu D, Li N, Yao X, Zhou L. Potential inflammatory markers in obstructive sleep apnea-hypopnea syndrome. Bosn J Basic Med Sci. 2017;17(1):47-53. doi:10.17305/bjbms.2016.1579

28. McArdle N, Hillman D, Beilin L, Watts G. Metabolic risk factors for vascular disease in obstructive sleep apnea: a matched controlled study. Am J Respir Crit Care Med. 2007;175(2):190-195. doi:10.1164/rccm.200602-270OC 
29. Newman AB, Nieto FJ, Guidry U, et al.; Sleep Herat Health Study Research Group. Relation of sleep-disordered breathing to cardiovascular disease risk factors: the Sleep Heart Health Study. Am Epidemiol. 2001;154(1):50-59. doi:10.1093/aje/154.1.50

30. Drager LF, Jun J, Polotsky VY. Obstructive sleep apnea and dyslipidemia: implications for atherosclerosis. Curr Opin Endocrinol Diabetes Obes. 2010;17(2):161-165. doi:10.1097/ MED.0b013e3283373624

31. Watts GF, Ooi EM, Chan DC. Therapeutic regulation of apoB100 metabolism in insulin resistance in vivo. Pharmacol Ther. 2009;123 (3):281-291. doi:10.1016/j.pharmthera.2009.04.005

32. Ozcelik F, Erdogan O, Aktoz M, Ekuklu G, Tatli E, Demir M. Diagnostic value of plasma fibronectin level in predicting the presence and severity of coronary artery disease. Ann Hematol. 2009;88 (33):249-253. doi:10.1007/s00277-008-0573-X

33. Hopps E, Canino B, Montana M, et al. Gelatinases and their tissue inhibitors in a group of subjects with obstructive sleep apnea syndrome. Clin Hemorheol Microcirc. 2016;62(1):27-34. doi: $10.3233 / \mathrm{CH}-151928$

34. Jurado-Gamez B, Gomez-Chaparro JL, Muñoz-Calero M, et al. Serum proteomic changes in adults with obstructive sleep apnoea. J Sleep Res. 2012;21(2):139-146. doi:10.1111/j.13652869.2011.00955.x
35. Carlson JT, Rangemark C, Hedner JA. Attenuated endothelium-dependent vascular relaxation in patients with sleep apnoea. J Hypertens. 1996;14(5):577-584. doi:10.1097/00004872199605000-00006

36. Wåhlin-Larsson B, Ulfberg J, Aulin KP, Kadi F. The expression of vascular endothelial growth factor in skeletal muscle of patients with sleep disorders. Muscle Nerve. 2009;40(4):556-561. doi:10.1002/ mus. 21357

37. Tan KC, Chow WS, Lam JC, et al. HDL dysfunction in obstructive sleep apnea. Atherosclerosis. 2006;184(2):377-382. doi:10.1016/j. atherosclerosis.2005.04.024

38. Wijelath ES, Murray J, Rahman S, et al. Novel vascular endothelial growth factor binding domains of fibronectin enhance vascular endothelial growth factor biological activity. Circ Res. 2002;91 (1):25-31. doi:10.1161/01.res.0000026420.22406.79

39. Palma JA, Urrestarazu E, Lopez-Azcarate J, et al. Increased sympathetic and decreased parasympathetic cardiac tone in patients with sleep related alveolar hypoventilation. Sleep. 2013;36(6):933-940. doi:10.5665/sleep. 2728

\section{Publish your work in this journal}

Nature and Science of Sleep is an international, peer-reviewed, open access journal covering all aspects of sleep science and sleep medicine, including the neurophysiology and functions of sleep, the genetics of sleep, sleep and society, biological rhythms, dreaming, sleep disorders and therapy, and strategies to optimize healthy sleep.
The manuscript management system is completely online and includes a very quick and fair peer-review system, which is all easy to use. Visit http://www.dovepress.com/testimonials.php to read real quotes from published authors. 